\title{
Population Pharmacokinetics of Ganciclovir after Valganciclovir Treatment in Children with Renal Transplant
}

\author{
A. Facchin,,$^{a, b, c}$ V. Elie, ${ }^{a}$ N. Benyoub, ${ }^{a}$ S. Magreault, ${ }^{a}$ A. Maisin, ${ }^{d}$ T. Storme, ${ }^{e}$ (D) W. Zhao, \\ aDépartement de Pharmacologie Pédiatrique et Pharmacogénétique, CHU Robert Debré, APHP, Paris, France \\ bService de Pharmacie, Centre Hospitalier Intercommunal Robert Ballanger, Aulnay-Sous-Bois, France \\ CUniversité de Paris, Ecole Doctorale MTCI, Paris, France \\ aService de Néphrologie Pédiatrique, Hôpital Robert Debré, APHP, Paris, France \\ eService de Pharmacie, CHU Robert Debré, APHP, Paris, France \\ fDepartment of Clinical Pharmacy, School of Pharmaceutical Sciences, Shandong University, Jinan, China \\ gPaediatric Research Institute, Children's Hospital of Hebei Province, Shijiazhuang, China \\ nUniversité de Paris, Paris, France
}

ABSTRACT Valganciclovir, the ganciclovir prodrug, is an antiviral agent administered orally to prevent or treat cytomegalovirus infection in solid-organ transplant recipients. The valganciclovir dosing regimen in children is still controversial, as the number of patients reaching the area under the concentration-time curve at steady state $\left(A U C_{s s}\right.$ ) target (40 to $60 \mathrm{mg} \cdot \mathrm{h} / \mathrm{liter}$ ) remains highly variable. The aim of this study was to determine the population pharmacokinetics of valganciclovir in pediatric renal transplant recipients and propose an appropriate dosing regimen. Children with renal transplant who received valganciclovir to prevent or treat cytomegalovirus infection at the Robert Debré University Hospital were included. Plasma ganciclovir concentrations were determined by high-performance liquid chromatography and UV detection. Population pharmacokinetic analysis was performed with NONMEM software. A total of 104 patients aged 2 to 20 years treated with valganciclovir, administered at a mean dose of $17.3 \pm 6.1 \mathrm{mg} / \mathrm{kg}$ of body weight to prevent and/or treat cytomegalovirus infection after renal transplantation, were included. A total of 1,212 samples were available. A two-compartment model with first-order elimination best fitted the data: ganciclovir clearance increased with body surface area, was 15\% higher in boys than in girls, and decreased with increasing creatinine concentration. The central volume of distribution increased with body surface area and was $14 \%$ higher in boys than in girls. According to the personalized dosing regimen, $65.7 \%$ and $65.4 \%$ of the children were predicted to achieve the $\mathrm{AUC}_{\mathrm{ss}}$ target for cytomegalovirus prophylaxis and treatment, respectively. A new pharmacokinetic model that allows the proposal of an individualized dose adapted to the characteristics of pediatric patients with renal transplant was built.

KEYWORDS cytomegalovirus, ganciclovir, pediatrics, population pharmacokinetics, renal transplantation

ytomegalovirus (CMV) infection is a major cause of morbidity in solid-organ transplant recipients. Valganciclovir, an antiviral agent, is commonly used to prevent or treat CMV infection in this population (1). Valganciclovir, the valine ester prodrug of ganciclovir, results in ganciclovir bioavailability $(F)$ of 55 to $60 \%$ in pediatric patients ( 2 , $3)$, up to 10 times higher than that of oral ganciclovir. Valganciclovir is rapidly converted to ganciclovir by liver and intestinal esterases and is predominantly eliminated unchanged by renal excretion (4). The indications for treatment with valganciclovir for the pediatric population of the Food and Drug Administration (FDA) include renal trans-
Citation Facchin A, Elie V, Benyoub N, Magreault S, Maisin A, Storme T, Zhao W, Deschenes G, Jacqz-Aigrain E. 2019. Population pharmacokinetics of ganciclovir after valganciclovir treatment in children with renal transplant. Antimicrob Agents Chemother 63:e01192-19. https://doi.org/10.1128/AAC .01192-19.

Copyright $\odot 2019$ American Society for Microbiology. All Rights Reserved. Address correspondence to E. Jacqz-Aigrain, evelyne.jacqzaigrain@gmail.com.

Received 12 July 2019

Returned for modification 5 August 2019

Accepted 3 September 2019

Accepted manuscript posted online 16

September 2019

Published 21 November 2019 
TABLE 1 Characteristics of the patients included in the pharmacokinetic analysis

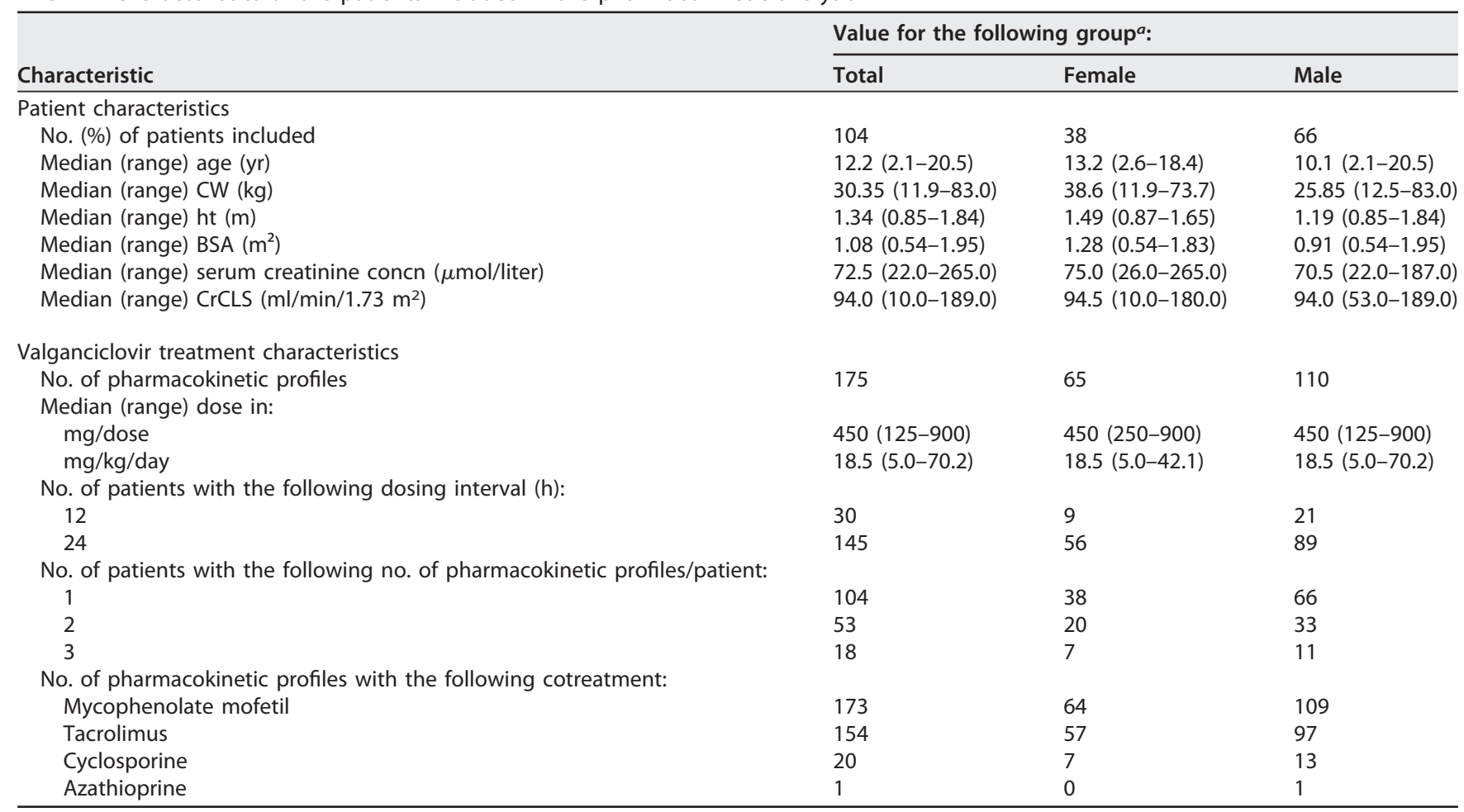

${ }^{a}$ Age, current body weight, height, body surface area, serum creatinine concentration, and creatinine clearance (Schwartz formula) (CrCLS) were not significantly different between female and male children (Wilcoxon test, $P>0.05$ ).

plantation in patients aged 4 months to 16 years and heart transplantation in patients aged 1 month to 16 years. However, valganciclovir is also used off-label to treat congenital CMV infection and to treat or prevent CMV or Epstein-Barr virus infection among other solid-organ and hematopoietic stem cell transplant patients in the pediatric population (5-7).

Different valganciclovir dosing regimens have been proposed, but the optimal dose is still controversial, as the percentage of patients reaching the pharmacological target remains variable (8). The use of subtherapeutic levels risks the emergence of CMV resistance (9) and clinical failure, whereas supratherapeutic levels increase the risk of adverse effects, such as leukopenia, neutropenia, and nephrotoxicity, the most relevant adverse effects in the pediatric population $(4,10)$. The objective of this study was to describe the valganciclovir pharmacokinetics in a large cohort of children with renal transplant and develop an optimized dosing regimen for this population.

\section{RESULTS}

Population characteristics and ganciclovir treatment. A total of 1,212 samples were collected from 104 patients aged 2 to 20 years treated with valganciclovir between August 1999 and December 2016, and from these, 175 pharmacokinetic profiles (treatment, $n=30$; prophylaxis, $n=145$ ) were obtained. Valganciclovir concentrations ranged from 0.25 to $22.23 \mathrm{ng} / \mathrm{ml}$. The patients' characteristics, biological parameters, valganciclovir treatment, and comedications are summarized in Table 1.

Valganciclovir was administered orally at a mean dose of $17.3 \pm 6.1 \mathrm{mg} / \mathrm{kg}$ of body weight (BW)/dose once a day (every $24 \mathrm{~h}$ [q24h]) or twice a day (every $12 \mathrm{~h}$ [q12h]), according to the indication (prophylaxis or treatment, respectively).

Model construction. A two-compartment model with first-order elimination and the following six parameters best fitted the data: absorption rate constant $\left(k_{a}\right)$, lag time (ALAG), apparent oral clearance $(\mathrm{CL} / F)$, apparent central volume of distribution $\left(V_{1} / F\right)$, apparent intercompartmental clearance $(Q / F)$, and apparent peripheral volume of 
TABLE 2 Covariate analysis ${ }^{a}$

\begin{tabular}{|c|c|c|c|c|c|c|c|}
\hline \multirow[b]{2}{*}{ Parameter } & \multirow{2}{*}{$\begin{array}{l}\text { Pharmacokinetic } \\
\text { parameter(s) }\end{array}$} & \multirow[b]{2}{*}{ OFV ( $\triangle$ OFV) } & \multicolumn{4}{|l|}{ IIV } & \multirow{2}{*}{$\begin{array}{l}\text { Residual } \\
\text { error (\% }\end{array}$} \\
\hline & & & $\mathrm{CL}$ & $V_{1}$ & $v_{2}$ & $k_{a}$ & \\
\hline Structural model & ALAG, $k_{a}, \mathrm{CL}, V_{1}, V_{2}, Q$ & $690.6(-22.3)$ & 35.4 & 41.2 & 106.7 & 101.5 & 33.3 \\
\hline \multicolumn{8}{|l|}{ Impact of covariates, forward selection } \\
\hline $\mathrm{BSA} / V_{1}$ & $v_{1}$ & $218.7(-177.9)$ & 31.8 & 12.4 & 37.4 & 56.4 & 23.3 \\
\hline $\mathrm{BSA} / V_{1}$ and $\mathrm{CrCLS} / \mathrm{CL}$ & $\mathrm{CL}, V_{1}$ & $171.6(-47.1)$ & 29.4 & 12.4 & 1.0 & 56.1 & 23.3 \\
\hline $\mathrm{BSA} / V_{1}, \mathrm{CrCLS} / \mathrm{CL}, \mathrm{CREA} / \mathrm{CL}, \mathrm{BSA} / \mathrm{CL}$, and gender $/ V_{1}$ & $\mathrm{CL}, \mathrm{V}_{1}$ & $61.4(-9.3)$ & 17.1 & 9.4 & 54.9 & 58.7 & 23.5 \\
\hline $\mathrm{BSA} / V_{1}, \mathrm{CrCLS} / \mathrm{CL}, \mathrm{CREA} / \mathrm{CL}, \mathrm{BSA} / \mathrm{CL}$, gender $/ V_{1}$ and gender $/ \mathrm{CL}$ & $\mathrm{CL}, V_{1}$ & $54.3(-7.1)$ & 16.0 & 9.3 & 54.6 & 59.2 & 23.5 \\
\hline $\begin{array}{l}\text { Final model, backward selection, } \mathrm{BSA} / V_{1}, \mathrm{CREA} / \mathrm{CL} \text { and } \mathrm{BSA} / \mathrm{CL} \text {, } \\
\text { gender } / V_{1} \text { and gender/Cl }\end{array}$ & $C L, V_{1}$ & $54.7(+0.4)$ & 16.0 & 9.3 & 54.6 & 59.2 & 23.5 \\
\hline \multicolumn{8}{|l|}{ Others models ${ }^{b}$} \\
\hline
\end{tabular}

${ }^{a} \mathrm{OFV}$, objective function value; $\triangle \mathrm{OFV}$, difference in OFV; IIV, interindividual variability; CREA, creatinine concentration; ALAG, lag time; $k_{a}$, absorption rate constant; CL, apparent clearance; $V_{1}$, apparent central volume of distribution; $V_{2}$, apparent peripheral volume of distribution; $Q$, apparent intercompartmental clearance.

${ }^{b}$ These 2 models showed that valganciclovir pharmacokinetics were better explained with BSA, creatinine concentration, and gender on $C L$ and $B S A$ and gender/ $V_{1}$ separately than when they were included in the CrCLS formula.

distribution $\left(V_{2} / F\right)$. Interindividual variability was best described by an exponential model, and residual variability was best described by a proportional model. Interindividual variability on $Q / F$ and ALAG did not improve the model. The interoccasion variability of $C L / F, k_{a}$ and $V_{2} / F$ improved the model significantly.

The allometric model was first tested and resulted in a significant drop in the objective function value (OFV) to 164.9. Body surface area (BSA) was the most significant parameter, impacting $V_{1} / F$ and allowing a drop in the OFV to 177.9 , and so the allometric model was not retained. Creatinine clearance on $\mathrm{CL} / F$ was then identified to be the most important covariate and associated with a significant drop in the OFV (difference in the OFV $[\triangle \mathrm{OFV}]=47.1$ ). BSA on $\mathrm{CL} / F$ also showed a significant drop in the OFV $(\triangle \mathrm{OFV}=74.1)$. $\mathrm{A}$ further decrease was observed with the implementation of the serum creatinine concentration on $\mathrm{CL} / F(\triangle \mathrm{OFV}=25)$. Finally, gender on $V_{1} / F$ and $\mathrm{CL} / F$ showed a significant drop in the OFV ( $\triangle \mathrm{OFV}=9.3$ and 7.1, respectively). The additional variables tested did not decrease the OFV significantly. After the backward elimination, creatinine clearance estimated by the Schwartz formula (CrCLS) did not remain significant $(\triangle \mathrm{OFV}=1.7)$, and three covariates (BSA, serum creatinine concentration, and gender) were retained after the backward elimination process. The results of the covariate analysis are presented in Table 2. Table 3 summarizes the parameter estimates of the final model. The estimated $C L / F$ was $11.3 \pm 3.1$ liters/h, the estimated mean volume of distribution at steady state (the sum of $V_{1}$ and $V_{2}$ ) was $75.1 \pm 23.9$ liters, the intercompartmental clearance was 1.5 liters/h, $k_{a}$ was $9.0 \pm 5.8 \mathrm{~h}^{-1}$, and ALAG was $0.86 \mathrm{~h}$. Apparent ganciclovir clearance increased with BSA, was $15 \%$ higher in boys than in girls, and decreased with increasing creatinine concentration. The central volume of distribution also increased with BSA and was $14 \%$ higher in boys than in girls.

Model evaluation. Model diagnostics showed an acceptable goodness of fit for the final model without a prediction bias: no trends in the plots of conditional weighted residuals (CWRES) and population predicted concentration (PRED) versus time were observed (Fig. 1). The median parameter estimates resulting from the bootstrap procedure closely agreed with the respective values from the final population model, indicating that the final model was stable and could redetermine the estimates of the population pharmacokinetic parameters. The results of 500 bootstrap replicates (a 93.4\% successful run) are summarized in Table 3. The distribution of the normalized prediction distribution error (NPDE) and histograms was consistent with the theoretical 
TABLE 3 Population pharmacokinetic parameters of valganciclovir and bootstrap results ${ }^{a}$

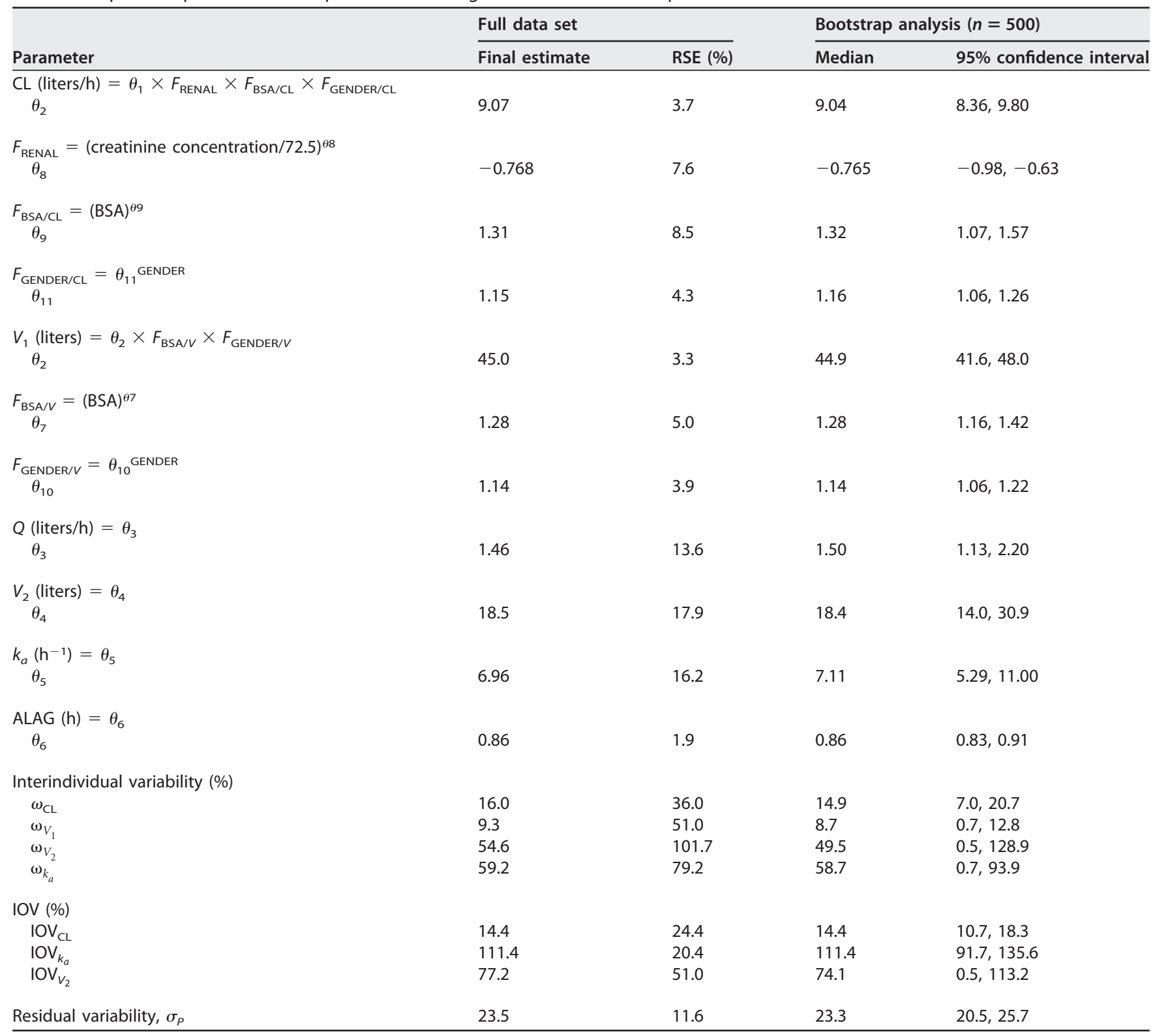

aRSE, relative standard error; IOV, interoccasion variability.

$N(0,1)$ distribution and density, indicating a good fit of the model to the individual data (Fig. 1). The mean and variance of the NPDE were 0.0381 (Wilcoxon signed-rank test, $P=0.402$ ) and 1.05 (Fisher variance test, $P=0.223$ ), respectively. The residuals also presented a normal distribution with no trend function for time or concentration.

The prediction-corrected visual predictive check (pc-VPC) showed that the predictioncorrected concentrations observed were consistent with the concentrations simulated by the model (Fig. 2).

Dosing regimen optimization. According to our current dosing regimen and rich pharmacokinetics ( $n=174$ ), only $42.5 \%$ of the values of the area under the concentrationtime curve at steady state over $24 \mathrm{~h}\left(\mathrm{AUC}_{\mathrm{ss}-24 \mathrm{~h}}\right)$ were within the therapeutic range for CMV prophylaxis, 30.8\% were over $60 \mathrm{mg} \cdot \mathrm{h} / \mathrm{liter}$, and $26.7 \%$ were under $40 \mathrm{mg} \cdot \mathrm{h} / \mathrm{liter}$ and only $34.5 \%$ of the values of the area under the curve at steady state over $12 \mathrm{~h}$ $\left(\mathrm{AUC}_{\mathrm{ss}-12 \mathrm{~h}}\right.$ ) were within the therapeutic range for CMV treatment, 20.7\% were over $60 \mathrm{mg} \cdot \mathrm{h} / \mathrm{liter}$, and $44.8 \%$ were under $40 \mathrm{mg} \cdot \mathrm{h} / \mathrm{liter}$. 
(a) DV vs PRED

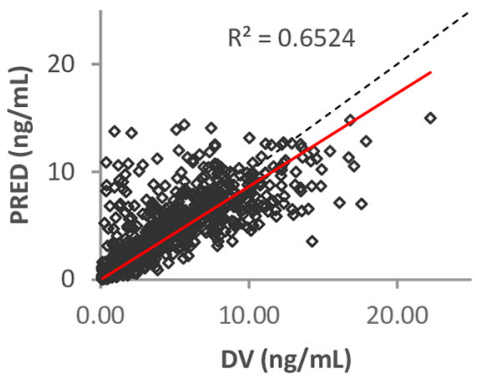

(c) CWRES vs PRED

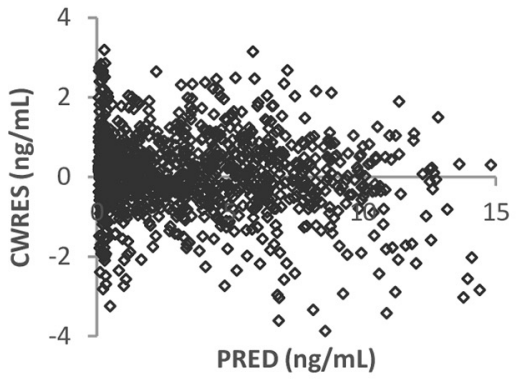

(e)

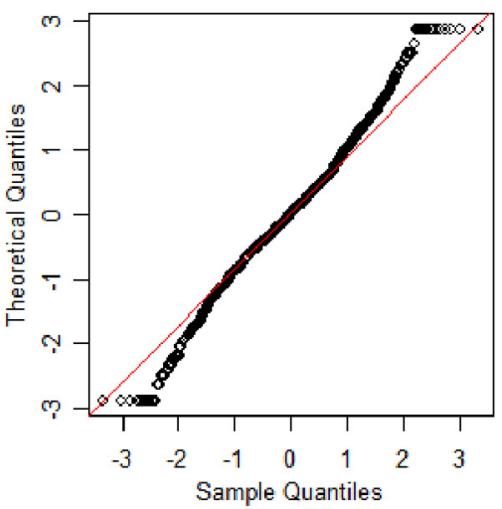

(g)

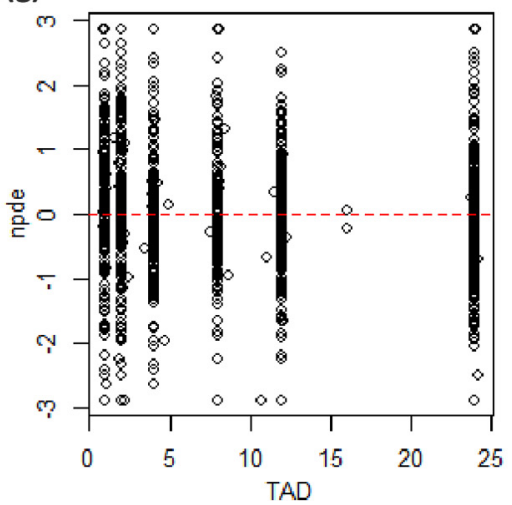

(b) DV vs IPRED

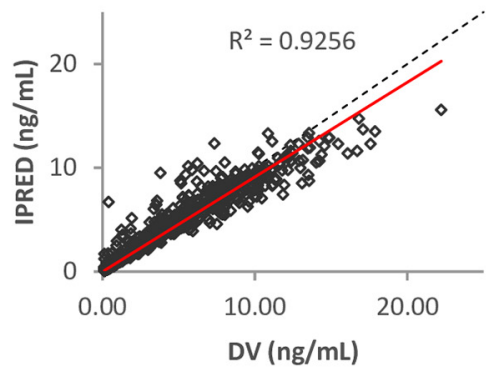

(d) CWRES vs Time

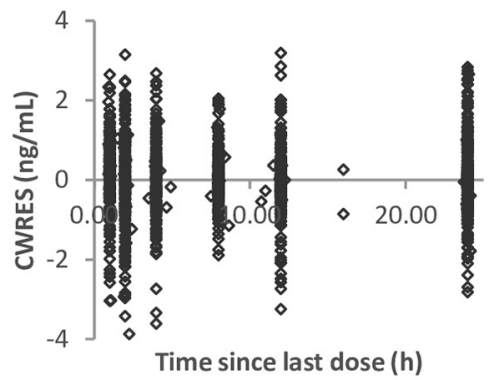

(f)

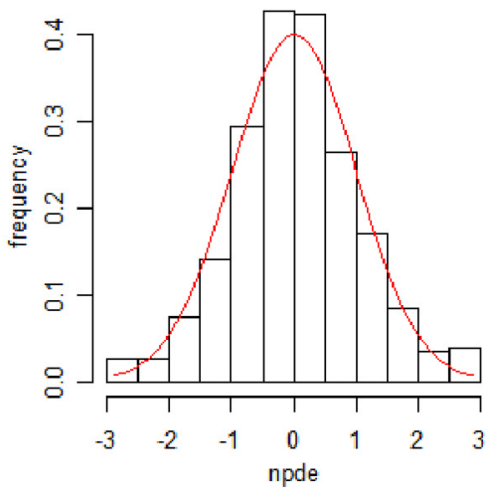

(h)

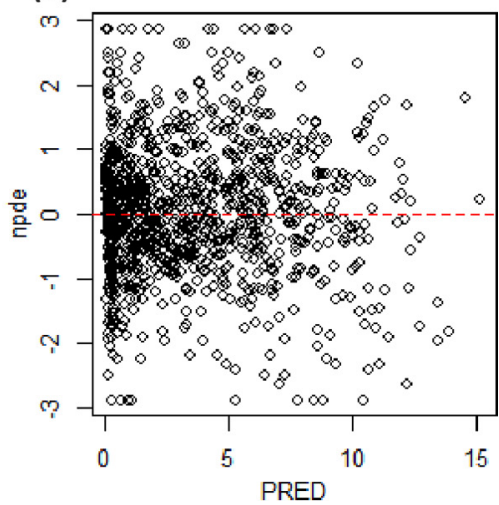

FIG 1 ( $a$ to $d$ ) Population predicted concentrations (PRED) versus observed concentrations (DV) (a), individual predicted concentrations (IPRED) versus DV (b), conditional weight residual concentrations (CWRES) versus PRED (c), and CWRES versus time since last dose (d). The dashed lines represent the lines of identity, and the solid red lines represent the linear regression lines. (e to h) QQ (quantilequantile) plot of the distribution of the normalized prediction distribution errors versus the theoretical distribution, $N(0,1)$ (represented by the red line) (e), histogram of the distribution of NPDE, with the density of the standard Gaussian distribution overlaid represented by the red line ( $f$ ), plot of NPDE versus time (TAD, time after last dose) (g), and plot of NPDE versus PRED (h). 


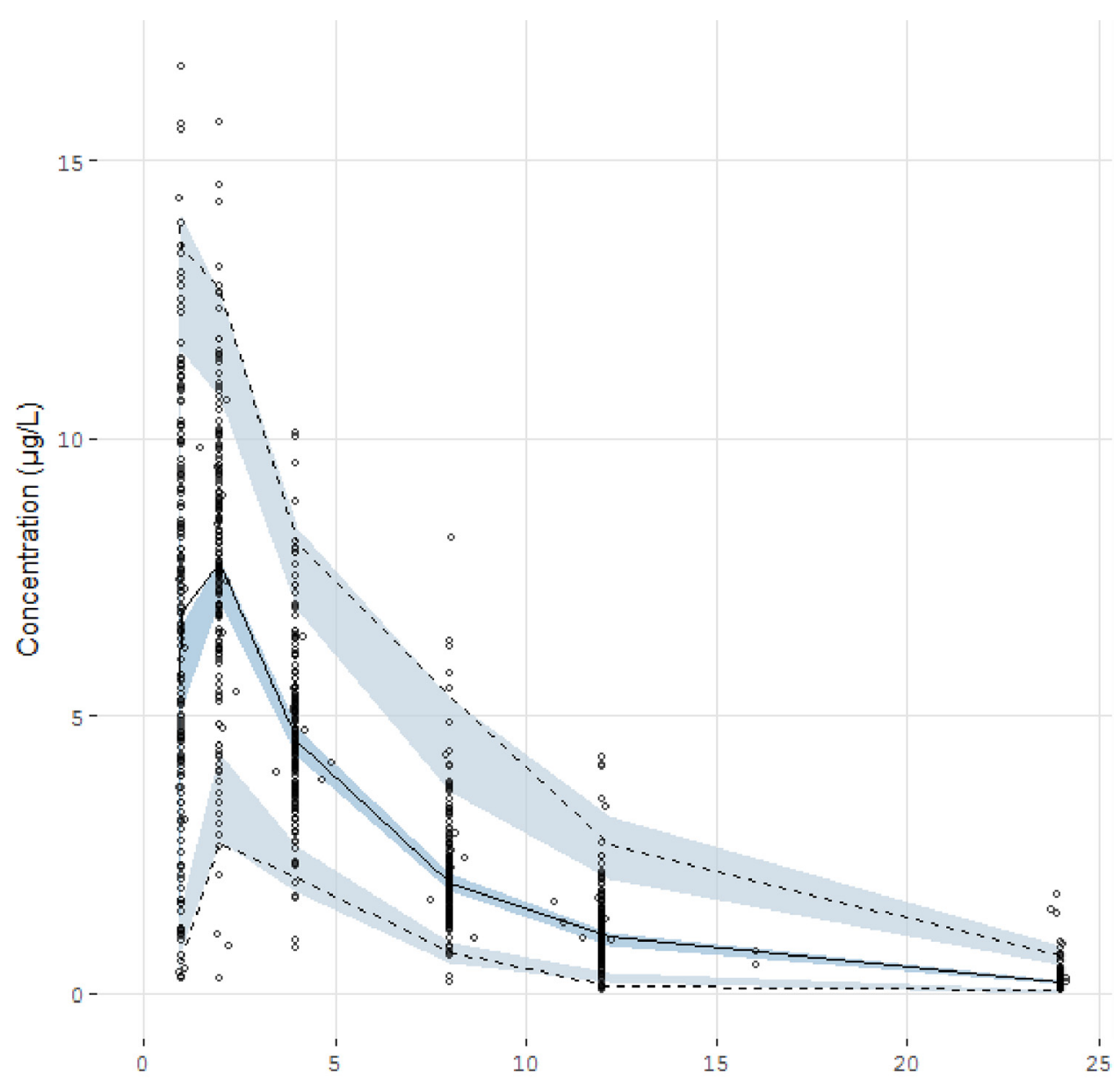

Time after last dose (h)

FIG 2 Prediction-corrected visual predictive check of the final model describing the pharmacokinetics of valganciclovir in children. The circles represent the prediction-corrected observed concentrations. The solid line represents the median of the prediction-corrected observed concentrations, and the semitransparent blue field represents the simulation-based $95 \%$ confidence intervals for the median. The observed 2.5th and 97.5th percentiles are indicated by dashed lines, and the $95 \%$ confidence intervals for the model-predicted percentiles are indicated as corresponding semitransparent blue fields.

Based on the final model, personalized optimized dosing was calculated with the following formulas: prophylactic valganciclovir dose (number of milligrams every 24 $\mathrm{h})=\mathrm{AUC}_{\mathrm{ss}-24 \mathrm{~h}}$ target $\times \mathrm{CL} / \mathrm{F}$ and therapeutic valganciclovir dose (number of milligrams every $12 \mathrm{~h})=\mathrm{AUC}_{\mathrm{ss}-12 \mathrm{~h}}$ target $\times \mathrm{CL} / F$, where clearance $(\mathrm{CL})$ was calculated using the equation developed from the model $\mathrm{CL} / F=9.07 \times$ (serum creatinine concentration/72.5) $)^{-0.768} \times \mathrm{BSA}^{1.31} \times 1.15^{\text {gender, }}$, with the serum creatinine concentration being expressed in micromoles per liter, BSA being expressed in square meters, and gender being fixed to 1 for male and 0 for female.

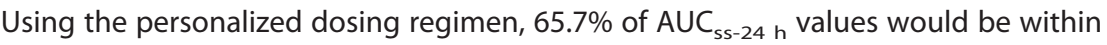
the therapeutic target for CMV prophylaxis, $19.7 \%$ would be over the therapeutic target range for CMV prophylaxis, and $14.6 \%$ would be under the therapeutic target range for CMV prophylaxis, while $65.4 \%$ of $\mathrm{AUC}_{\mathrm{ss}-12 \mathrm{~h}}$ values would be satisfactory for CMV treatment, $14.5 \%$ would be over $60 \mathrm{mg} \cdot \mathrm{h} / \mathrm{liter}$, and $20.1 \%$ would be under $40 \mathrm{mg} \cdot \mathrm{h} / \mathrm{liter}$.

\section{DISCUSSION}

This work successfully described the valganciclovir population pharmacokinetics in a large cohort of children with renal transplant. Valganciclovir pharmacokinetics were better described by a two-compartment model with first-order elimination and were influenced by BSA and the serum creatinine concentration, and, for the first time in 
children, a gender effect on clearance and the central volume of distribution was observed.

Valganciclovir, the prodrug of ganciclovir, is administered orally to prevent and treat CMV infection in renal transplant recipients (4). Its bioavailability is much higher than that of ganciclovir (11), but the pharmacokinetic variability of the ganciclovir disposition is high.

In the present study, the mean of the weight-normalized estimated clearance $(0.39 \pm 0.14 \mathrm{liter} / \mathrm{h} / \mathrm{kg})$ was comparable to the values previously reported in adults $(0.32$ liter $/ \mathrm{h} / \mathrm{kg}$ ) (12) and in children (0.30 liter/h/ $\mathrm{kg} /$ ) (13). Monitoring of ganciclovir on the basis of the area under the concentration-time curve is recommended in both adult and pediatric solid-organ transplant patients (14), with the target being an $A U C_{s s}$ between two administrations (12 or $24 \mathrm{~h}$ ) and at steady state of between 40 and $60 \mathrm{mg} \cdot \mathrm{h} / \mathrm{liter}(14,15)$.

Different pharmacokinetic models describing ganciclovir pharmacokinetics have been published, and different dosing algorithms have been tested in adults and children, but the optimal dosing regimen remains controversial. In children in different age groups and with different indications for treatment, BSA, CW, and/or renal clearance has been identified to be a covariate explaining part of the observed variability ( 3 , $8,10,13,15,16)$. Our first population pharmacokinetic study in 22 children with renal transplant identified that current weight and creatinine clearance, two factors that were included in the proposed dosing regimen, influenced ganciclovir clearance (13). The pharmacokinetic model by Vaudry and coauthors (8) suggested that the individual dose be calculated with the following equation: dose $=7 \times \mathrm{BSA} \times \mathrm{CrCLS}$, where the dose is in milligrams and BSA is in square meters. However, this formula was associated with high exposures and toxicity in children with renal transplant with a low current weight and normal renal function, as pointed out by the FDA in 2010 (17). Pescovitz et al. revealed the influence of age on the valganciclovir apparent $A \cup C_{s s}$ and $C L$, with bioavailability being lower in the youngest children (3). They recommended that doses be adjusted according to both BSA and renal function and that $100 \%, 50 \%$, and $25 \%$ of a $520-\mathrm{mg} / \mathrm{m}^{2}$ dose be given when renal clearance was over 70 , between 50 and 69 , or between 40 and $49 \mathrm{ml} / \mathrm{min}$, respectively (3). As a result, with a pediatric dosing regimen derived from the adult dose and adjusted to renal function, the youngest patients (age, $<5$ years) were underexposed (4). Moreover, information on children with poor renal function is lacking, as they were excluded from the study. Åsberg et al. proposed a different population-based dosing algorithm, depending on CW and estimated clearance calculated by the Cockcroft and Gault equation (GFR): dose (in milligrams) = weight $\times(0.07 \times \mathrm{GFR}+k)$ (where $k$ is a correction factor for renal function), but this was still not optimal in patients with extreme renal function or CW (15). Additional observational studies that have tested manufacturer doses and different dosing regimen, that have reported AUCs in small groups of patients (18-20), and that have shown that the target $A_{U S}$ for ganciclovir was achieved only in a limited number of patients are available.

Dosing simulations with the published models tested different dosing regimens, but the percentage of patients reaching the pharmacologically desired ganciclovir exposure was, at most, $30 \%(15,19,20)$ and was $40 \%$ in the Robert Debré University Hospital prior to the present study. In contrast, by individualizing valganciclovir doses, $65.7 \%$ and $65.4 \%$ of $A U C_{s s}$ values were within the therapeutic range for CMV infection prophylaxis and treatment, respectively, and are much higher than those achieved in previous studies. Moreover, as $V_{1}$ and apparent $\mathrm{CL}$ are higher in boys than in girls, boys would need higher doses than girls to reach the AUC target. Such an influence of gender on valganciclovir pharmacokinetics was already described in adults (21) but was not described in children. Since ganciclovir is partly secreted by the kidney, this may result from gender differences in organic anion transporter expression and maturation (22).

Our study has some weaknesses, as the model was built only with ganciclovir concentrations following oral valganciclovir administration and bioavailability could 
not be calculated. However, this parameter showed low variability in children, ranging from $55 \%$ to $60 \%(3,11,15)$.

Conclusion. The ganciclovir pharmacokinetic model developed in children with renal transplant receiving valganciclovir revealed that its apparent clearance is higher in boys than in girls, increases with BSA, and decreases with the serum creatinine concentration. The new dosing regimen would allow two-thirds of children to reach the pharmacological target but will need to be validated prospectively.

\section{MATERIALS AND METHODS}

Study population. Pediatric renal transplant recipients receiving valganciclovir to prevent or treat CMV infection at the Robert Debré University Hospital were included in this retrospective analysis. Only patients with pharmacokinetic samples drawn during routine valganciclovir therapeutic drug monitoring were selected. The research was conducted in accordance with the recommendations of the institutional research committee (Comité de l'Evaluation de l'Ethique des Projets de Recherche Biomédicale de Robert Debré CEERB, no. 221), the 1964 Helsinki Declaration and its later amendments, and the French good clinical practice guidelines. No consent was required for this type of retrospective study. All patients received an induction therapy with antilymphocyte serum or basiliximab and were treated with prednisone, azathioprine, or mycophenolate mofetil (MMF)-mycophenolic acid (MPA) and tacrolimus or cyclosporine after transplantation.

Valganciclovir administration, therapeutic drug monitoring, and pharmacokinetic sampling. Valganciclovir was administered orally once a day (q24h; for prevention) or twice a day (q12h; for infection). Plasma samples for clinically indicated therapeutic drug monitoring were drawn at $1 \mathrm{~h}, 2 \mathrm{~h}, 4$ $\mathrm{h}, 8 \mathrm{~h}, 12 \mathrm{~h}$, and/or $24 \mathrm{~h}$ after valganciclovir administration, depending on the dosing regimen. A sample was obtained at $24 \mathrm{~h}$ when valganciclovir was administered once daily. The dosing recommendations in the Robert Debré University Hospital are dependent on the child's age and creatinine clearance estimated by the Schwartz formula (CrCLS) (23).

The valganciclovir dosing regimen for CMV prophylaxis depends on age: (i) for children less than 12 years of age, the recommended dose is calculated as dose (in milligrams) $=7 \times$ body surface area $(B S A) \times C r C L S$ (in milliliters per minute per $1.73 \mathrm{~m}^{2}$ ) once a day, with an upper limit of $150 \mathrm{ml} / \mathrm{min} / 1.73$ $\mathrm{m}^{2}$ for CrCLS and with BSA estimated by Mosteller's equation \{BSA (in square meters) $=$ [height (in centimeters) $\times$ weight (in kilograms)/3,600 $]^{0.53}(8)$, and (ii) in children over 12 years of age, the recommended dose is adjusted according to CrCLS $(900 \mathrm{mg} / 24 \mathrm{~h}, 450 \mathrm{mg} / 24 \mathrm{~h}, 450 \mathrm{mg} / 48 \mathrm{~h}$, and $450 \mathrm{mg} / 72 \mathrm{~h}$ for those with a CrCLS over $60 \mathrm{ml} / \mathrm{min} / 1.73 \mathrm{~m}^{2}, 40$ to $59 \mathrm{ml} / \mathrm{min} / 1.73 \mathrm{~m}^{2}, 25$ to $39 \mathrm{ml} / \mathrm{min} / 1.73 \mathrm{~m}^{2}$, and 10 to $25 \mathrm{ml} / \mathrm{min} / 1.73 \mathrm{~m}^{2}$, respectively) and is contraindicated in those with a CrCLS below $10 \mathrm{ml} / \mathrm{min} /$ $1.73 \mathrm{~m}^{2}$. For the treatment of CMV infection, the same doses are given twice daily when CrCLS is over $40 \mathrm{ml} / \mathrm{min} / 1.73 \mathrm{~m}^{2}$, once daily when CrCLS is between 25 and $39 \mathrm{ml} / \mathrm{min} / 1.73 \mathrm{~m}^{2}$, and every $48 \mathrm{~h}$ when $\mathrm{CrCLS}$ is under $25 \mathrm{ml} / \mathrm{min} / 1.73 \mathrm{~m}^{2}$. The ganciclovir area under the concentration-time curve at steady state $\left(A \cup C_{s s}\right.$ ) target was between 40 amd $60 \mathrm{mg} \cdot \mathrm{h} /$ liter.

Data on dose administration (e.g., start date, route, time of sampling, age, weight, gender) were available on the monitoring form requested for therapeutic drug monitoring. Additional patient characteristics and associated therapies were collected retrospectively from the medical charts: gender, height, serum creatinine concentration, proteinuria, underlying disease, indication for treatment (CMV infection or prophylaxis), and associated therapies.

Analytical assay method for ganciclovir. The analytical method for ganciclovir was adapted from the method described by Zhao et al. (13). Plasma concentrations were determined using highperformance liquid chromatography (HPLC) with UV detection at $254 \mathrm{~nm}$. The calibration curve for ganciclovir ranged from 0.25 to $25 \mathrm{mg} / \mathrm{ml}(0.25,0.5,1,5,10$, and $25 \mathrm{mg} / \mathrm{ml})$. The intraday coefficients of variation (CVs) were between $0.5 \%$ and $2.3 \%$ for calibration concentrations, and the interday CVs were $6.25 \%$ for calibration and $3.3 \%$ for the controls. The lower limit of quantification (LLOQ) was $0.25 \mathrm{mg} / \mathrm{ml}$. Serum creatinine concentrations were measured by the Jaffé method using the Architect $C$ system (Abbott Diagnostics, IL, USA).

Population pharmacokinetic analysis. Data were imported into R or Excel software for exploratory analysis and formatted for subsequent modeling. Pharmacokinetic analysis was carried out using the nonlinear mixed effects modeling program NONMEM (v7.2; Icon Development Solutions, Ellicott City, $M D, U S A$ ) with the gfortran (v4.6.0) compiler. Oral doses of valganciclovir were administered into a depot compartment. The absorption of valganciclovir was modeled as a first-order process, and conversion to ganciclovir in the central compartment was assumed to be instantaneous. A first-order conditional estimation (FOCE) method with an interaction option was used to estimate the pharmacokinetic parameters and their variability. One- and two-compartment models with first-order elimination were tested. The two-compartment model aimed to estimate the absorption rate constant $\left(k_{a}\right)$, apparent oral clearance $(C L / F)$, apparent central volume of distribution $\left(V_{1} / F\right)$, apparent peripheral volume of distribution $\left(V_{2} / F\right)$, apparent intercompartmental clearance $(Q / F)$, and lag time (ALAG) using the ADVAN subroutines.

Interindividual variability was best described with an exponential model and was expressed as follows: $\theta_{i}=\theta_{\text {mean }} \times e^{\eta_{i}}$, where $\theta_{i}$ represents the parameter value of the ith subject, $\theta_{\text {mean }}$ is the typical value of the parameter in the population, and $\eta_{i}$ is the variability between subjects, which is assumed to 
follow a normal distribution with a mean of zero and a variance of $\omega^{2}$. To find the most appropriate residual error model, additive, proportional, and mixed error forms were tested.

Covariate analysis. The following continuous and categorical covariates were tested: demographic covariates (current body weight [CW], height, age, BSA, and gender), clinical covariates (comedications and underlying disease), and biological covariates (serum creatinine concentration, creatinine clearance, serum uremia, and proteinuria). Results are reported as the mean and standard deviation for continuous variables and number and percentage for categorial variables, with a $P$ value of $<0.05$ being considered statistically significant.

Covariate analysis followed a forward and backward selection process.

The likelihood ratio test was used to test the effect of each variable on the model parameters. During the first step of covariate model building, a covariate was included if a significant decrease $\left(P<0.05, \chi^{2}\right.$ distribution with 1 degree of freedom, reduction $>3.84$ ) in the objective function value (OFV) from the basic model was obtained. All the significant covariates were then added simultaneously into a full model. Subsequently, each covariate was independently removed from the full model. If the increase in the OFV was higher than 6.635 ( $P<0.01, \chi^{2}$ distribution), the covariate was considered significantly associated with the pharmacokinetic parameter and was therefore retained in the final model.

The allometric size approach was tested by incorporating the $\mathrm{CW}$ into the basic model (a coefficient of 1 for $V_{1} / F$ and $V_{2} / F$ and a coefficient of 0.75 for $C L / F$ and $Q / F$ ).

Model validation. Validation of the final model was based on graphical and statistical methods. The goodness of fit was assessed by the population predicted concentration (PRED) and individual predicted concentration (IPRED) versus the observed concentration (DV) and conditional weighted residuals (CWRES) versus PRED and time. A prediction-corrected visual predictive check (pc-VPC) and normalized prediction distribution errors (NPDE) were performed by using simulated concentrations of 500 virtual data sets with the final population model parameters. Then, the stability and performance of the final model were also assessed by means of a nonparametric bootstrap analysis with resampling and replacement. Resampling was repeated 500 times, and the values of the estimated parameters from the bootstrap procedure were compared with those estimated from the original data set. The 2.5th and 97.5th percentiles of the median population pharmacokinetic parameters were regarded as the lower and upper $95 \%$ confidence interval limits, respectively.

Dosing regimen optimization. The parameter estimates obtained from the model were used for valganciclovir dosing optimization. Five hundred simulated trials were performed using NONMEM. The simulation cohort was built with all the patients from the original data set. As the recommend $A U C_{s s}$ is between 40 and $60 \mathrm{mg} \cdot \mathrm{h} /$ liter over $24 \mathrm{~h}$ for prevention and over $12 \mathrm{~h}$ for treatment (14), the $A U C_{s s}$ value of $50 \mathrm{mg} \cdot \mathrm{h} / \mathrm{liter}$ was selected for both prevention $\left(A \cup C_{s s-24 h}\right)$ and treatment $\left(A \cup C_{s s-12 h}\right)(14,15)$.

\section{ACKNOWLEDGMENTS}

We thank all the nurses who took care of the patients in the Department of Pediatric Nephrology, Robert Debré University Hospital, and the technicians who performed the biochemical analysis in the Department of Pediatric Pharmacology, Robert Debré University Hospital.

A.F., V.E., N.B., and E.J.-A. planned the study. A.M. and G.D. were in charge of patient care after renal transplantation. S.M. and T.S. were in charge of bioanalysis and pharmacological follow-up. A.F. conducted the pharmacokinetic analysis under the supervision of W.Z. and E.J.-A. A.F. wrote the first version of the manuscript with the help of E.J.-A. All authors revised and accepted the final and revised versions.

This research received no specific grant from any funding agency in the public, commercial, or not-for-profit sectors.

The Children's Hospital of Hebei Province is affiliated with Hebei Medical University. A. Facchin, V. Elie, N. Benyoub, S. Magreault, A. Maisin, T. Storme, W. Zhao, G. Deschenes, and E. Jacqz-Aigrain have no conflict of interet to declare.

\section{REFERENCES}

1. Humar A, Snydman D, AST Infectious Diseases Community of Practice. 2009. Cytomegalovirus in solid organ transplant recipients. Am J Transplant 9(Suppl 4):S78-S86. https://doi.org/10.1111/j.1600-6143 2009.02897.x.

2. Asberg A, Humar A, Rollag $H$, Jardine AG, Mouas $H$, Pescovitz MD, Sgarabotto D, Tuncer M, Noronha IL, Hartmann A, VICTOR Study Group. 2007. Oral valganciclovir is noninferior to intravenous ganciclovir for the treatment of cytomegalovirus disease in solid organ transplant recipients. Am J Transplant 7:2106-2113. https://doi.org/10.1111/j.1600-6143 2007.01910.x.

3. Pescovitz MD, Ettenger RB, Strife CF, Sherbotie JR, Thomas SE, McDiarmid S, Bartosh S, Ives J, Bouw MR, Bucuvalas J. 2009. Pharmacokinetics of oral valganciclovir solution and intravenous ganciclovir in pediatric renal and liver transplant recipients. Transpl Infect Dis 12:195-203. https://doi.org/10.1111/j.1399-3062.2009.00478.x.

4. Yu MA, Park JM. 2013. Valganciclovir: therapeutic role in pediatric solid organ transplant recipients. Expert Opin Pharmacother 14:807-815. https://doi.org/10.1517/14656566.2013.778244.

5. Meine Jansen CF, Toet MC, Rademaker CMA, Ververs TFF, Gerards LJ, van Loon AM. 2005. Treatment of symptomatic congenital cytomegalovirus infection with valganciclovir. J Perinat Med 33:364-366.

6. Schulzke S, Bührer C. 2006. Valganciclovir for treatment of congenital cytomegalovirus infection. Eur J Pediatr 165:575-576. https://doi.org/10 .1007/s00431-006-0109-0.

7. Müller A, Eis-Hübinger AM, Brandhorst G, Heep A, Bartmann P, Franz AR. 2008. Oral valganciclovir for symptomatic congenital cytomegalovirus 
infection in an extremely low birth weight infant. J Perinatol 28:74-76. https://doi.org/10.1038/sj.jp.7211854.

8. Vaudry W, Ettenger R, Jara P, Varela-Fascinetto G, Bouw MR, Ives J, Walker R, Valcyte WV16726 Study Group. 2009. Valganciclovir dosing according to body surface area and renal function in pediatric solid organ transplant recipients. Am J Transplant 9:636-643. https://doi.org/ 10.1111/j.1600-6143.2008.02528.x.

9. Razonable RR. 2018. Drug-resistant cytomegalovirus: clinical implications of specific mutations. Curr Opin Organ Transplant 23:388-394. https://doi.org/10.1097/MOT.0000000000000541.

10. Stockmann C, Roberts JK, Knackstedt ED, Spigarelli MG, Sherwin CM. 2015. Clinical pharmacokinetics and pharmacodynamics of ganciclovir and valganciclovir in children with cytomegalovirus infection. Expert Opin Drug Metab Toxicol 11:205-219. https://doi.org/10.1517/17425255 .2015.988139.

11. Pescovitz MD, Rabkin J, Merion RM, Paya CV, Pirsch J, Freeman RB, O'Grady J, Robinson C, To Z, Wren K, Banken L, Buhles W, Brown F. 2000. Valganciclovir results in improved oral absorption of ganciclovir in liver transplant recipients. Antimicrob Agents Chemother 44:2811-2815. https://doi.org/10.1128/AAC.44.10.2811-2815.2000.

12. Czock D, Scholle C, Rasche FM, Schaarschmidt D, Keller F. 2002. Pharmacokinetics of valganciclovir and ganciclovir in renal impairment. Clin Pharmacol Ther 72:142-150. https://doi.org/10.1067/mcp.2002.126306.

13. Zhao W, Baudouin V, Zhang D, Deschênes G, Le Guellec C, Jacqz-Aigrain E. 2009. Population pharmacokinetics of ganciclovir following administration of valganciclovir in paediatric renal transplant patients. Clin Pharmacokinet 48:321-328. https://doi.org/10.2165/00003088-200948050-00004.

14. Wiltshire H, Paya CV, Pescovitz MD, Humar A, Dominguez E, Washburn K, Blumberg E, Alexander B, Freeman R, Heaton N, Zuideveld KP, Valganciclovir Solid Organ Transplant Study Group. 2005. Pharmacodynamics of oral ganciclovir and valganciclovir in solid organ transplant recipients. Transplantation 79:1477-1483. https://doi.org/10 1097/01.TP.0000164512.99703.AD.

15. Åsberg A, Bjerre A, Neely M. 2014. New algorithm for valganciclovir dosing in pediatric solid organ transplant recipients. Pediatr Transplant 18:103-111. https://doi.org/10.1111/petr.12179.
16. Vezina HE, Brundage RC, Balfour HH. 2014. Population pharmacokinetics of valganciclovir prophylaxis in paediatric and adult solid organ transplant recipients. Br J Clin Pharmacol 78:343-352. https://doi.org/10 $.1111 / \mathrm{bcp} .12343$.

17. Center for Drug Evaluation and Research, Food and Drug Administration. 2010. FDA drug safety communication: new dosing recommendations to prevent potential Valcyte (valganciclovir) overdose in pediatric transplant patients. https://www.fda.gov/Drugs/DrugSafety/ucm225727 .htm.

18. Launay E, Théôret $Y$, Litalien C, Duval M, Alvarez F, Lapeyraque A-L, Phan V, Larocque D, Poirier N, Lamarre V, Ovetchkine P. 2012. Pharmacokinetic profile of valganciclovir in pediatric transplant recipients. Pediatr Infect Dis J 31:405-407. https://doi.org/10.1097/INF.0b013e3182463a19.

19. Villeneuve D, Brothers A, Harvey E, Kemna M, Law Y, Nemeth T, Gantt S. 2013. Valganciclovir dosing using area under the curve calculations in pediatric solid organ transplant recipients. Pediatr Transplant 17:80-85. https://doi.org/10.1111/petr.12030.

20. Peled O, Berkovitch M, Rom E, Bilavsky E, Bernfeld Y, Dorfman L, Pappo A, Ziv-Baran T, Brandriss N, Bar-Haim A, Amir J, Ashkenazi-Hoffnung L. 2017. Valganciclovir dosing for cytomegalovirus prophylaxis in pediatric solidorgan transplant recipients: a prospective pharmacokinetic study. Pediatr Infect Dis J 36:745-750. https://doi.org/10.1097/INF.0000000000001595.

21. Perrottet N, Csajka C, Pascual M, Manuel O, Lamoth F, Meylan P, Aubert JD, Venetz JP, Soccal P, Decosterd LA, Biollaz J, Buclin T. 2009. Population pharmacokinetics of ganciclovir in solid-organ transplant recipients receiving oral valganciclovir. Antimicrob Agents Chemother 53: 3017-3023. https://doi.org/10.1128/AAC.00836-08.

22. Nigam SK, Bush KT, Martovetsky G, Ahn S-Y, Liu HC, Richard E, Bhatnagar V, Wu W. 2015. The organic anion transporter (OAT) family: a systems biology perspective. Physiol Rev 95:83-123. https://doi.org/10.1152/ physrev.00025.2013.

23. Schwartz GJ, Brion LP, Spitzer A. 1987. The use of plasma creatinine concentration for estimating glomerular filtration rate in infants, children, and adolescents. Pediatr Clin North Am 34:571-590. https://doi .org/10.1016/s0031-3955(16)36251-4. 